Department of Ophthalmology and Orthoptics

University of Sheffield

Royal Hallamshire Hospital

Glossop Road Sheffield S10 2JF, UK

Sir,

\section{Fundal findings preceding retinal artery macroaneurysm}

Retinal artery macroaneurysms typically affect the first three divisions of the central retinal artery in one eye of elderly hypertensive women. ${ }^{1,2}$ Although the natural history of retinal artery macroaneurysms after first detection is well documented, ${ }^{2-5}$ only two previous reports have commented on fundal findings prior to the development of a macroaneurysm. ${ }^{4,6}$ We present a case report of the fundal findings in a patient with documented retinal artery macroaneurysm in one eye with subsequent development of another retinal artery macroaneurysm in her second eye.

\section{Case report}

Clinical review of a 78-year-old well-controlled hypertensive woman 10 weeks after laser treatment for a left retinal artery macroaneurysm revealed the development of a second arterial macroaneurysm in her right eye. Initial visual acuity in the right eye had been $6 /$ 12 but was reduced at this presentation to $6 / 24$. Fundal examination demonstrated a retinal artery macroaneurysm at the first bifurcation of the superotemporal retinal artery associated with pre-retinal and intra-retinal haemorrhage and macular oedema confirmed by fluorescein angiography (Figs. 1A, 2A). In addition there was macular exudate, arteriovenous nipping and hyaline vascular change consistent with hypertensive retinopathy (Fig. 1A). Review of colour

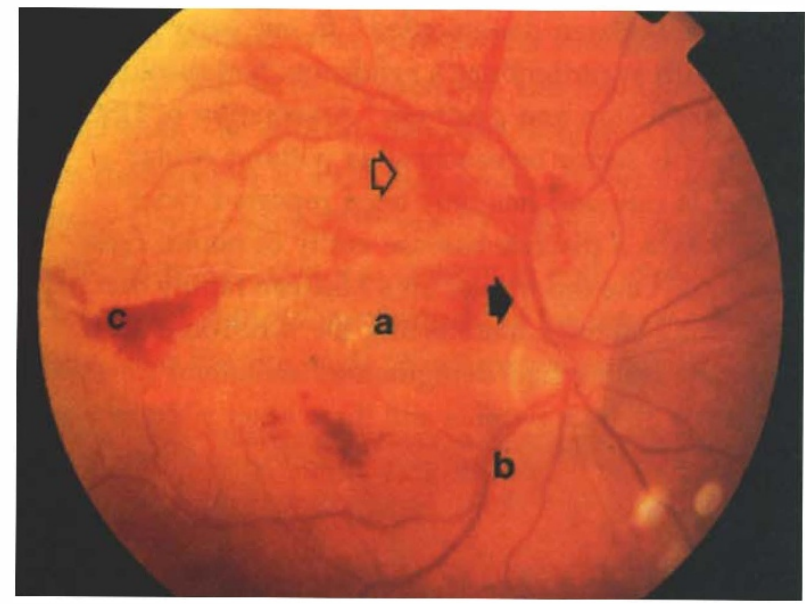

(A) fundal photographs taken 10 weeks earlier demonstrated pre-existent macular exudate, arteriovenous nipping and hyaline vascular change with mild focal dilatations of the retinal arteries (Fig. 1B). The point where the macroaneurysm arose corresponded to one of these focal dilatations at the first bifurcation of the superotemporal retinal artery (Fig. 1B). Previous fluorescein angiography did not demonstrate pre-existent macroaneurysm or any areas of fluorescein leakage in the right eye (Fig. 2B). The macroaneurysm was treated with focal argon laser photocoagulation and visual acuity at 4 month follow-up was $6 / 18$.

\section{Comment}

Retinal artery macroaneurysms are acquired dilatations of the first three orders of the retinal arteries that typically occur unilaterally in elderly hypertensive women. ${ }^{1-5}$ As in this report two previous reports have demonstrated retinal arterial macroaneurysms developing on a background of generalised retinal artery damage characterised by pre-existent retinal exudate, generalised arteriovenous nipping and hyaline vascular change. ${ }^{4,6}$ This report highlights that in the presence of generalised retinal damage, focal retinal arterial dilatation may be present and can progress to retinal artery macroaneurysm with associated haemorrhage and oedema in a matter of weeks. These clinical signs are supported by histological findings in retinal arteries of older people including thickening of the vessel walls and replacement of contractile elements with collagen. ${ }^{7}$ The actual site of the macroaneurysm may be related to focal areas of damage such as previous occlusion secondary to a retinal embolus or areas of turbulent blood flow such as at arterial bifurcations. ${ }^{4,6}$ The documented preponderance of retinal arterial macroaneurysms in the

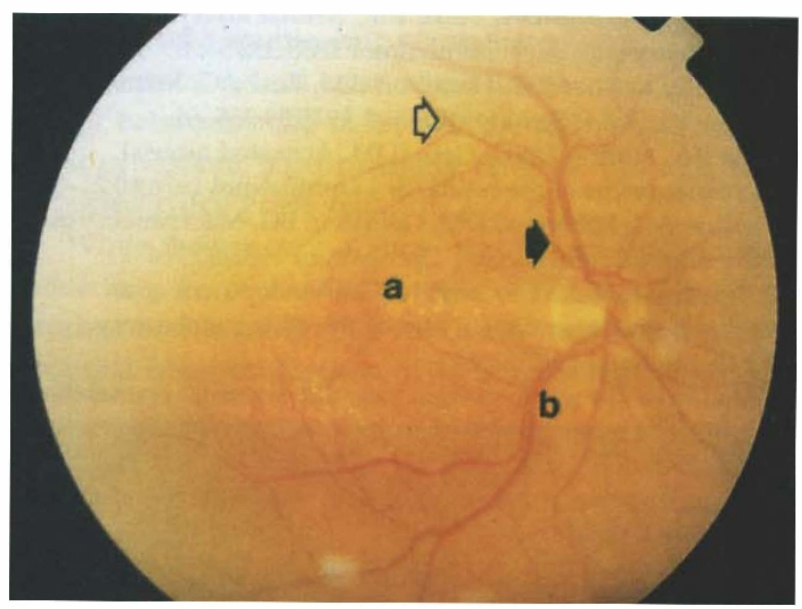

(B)

Fig. 1. (A) Colour photograph of the right fundus demonstrating a retinal artery macroaneurysm arising from the first bifurcation of the superotemporal retinal artery partially obscured by intra-retinal haemorrhage (open arrow). There is also evidence of macular exudate (a), arteriovenous nipping $(b)$ and hyaline vascular change (filled arrow) consistent with hypertensive retinopathy and pre-retinal haemorrhage (c). (B) Colour photograph of the right fundus taken 10 weeks preceding that in $(A)$, demonstrating mild dilatation of retinal arteries one of which corresponds to the point of origin of the retinal artery macroaneurysm at the first bifurcation of the superotemporal retinal artery (open arrow). There is also pre-existent macular exudate (a), arteriovenous nipping $(b)$ and hyaline vascular change (filled arrow). 


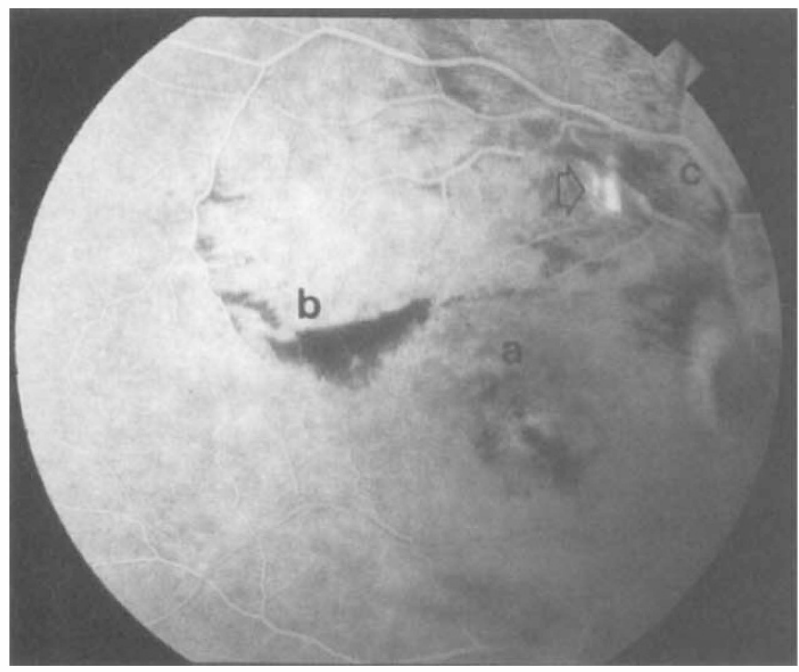

(A)

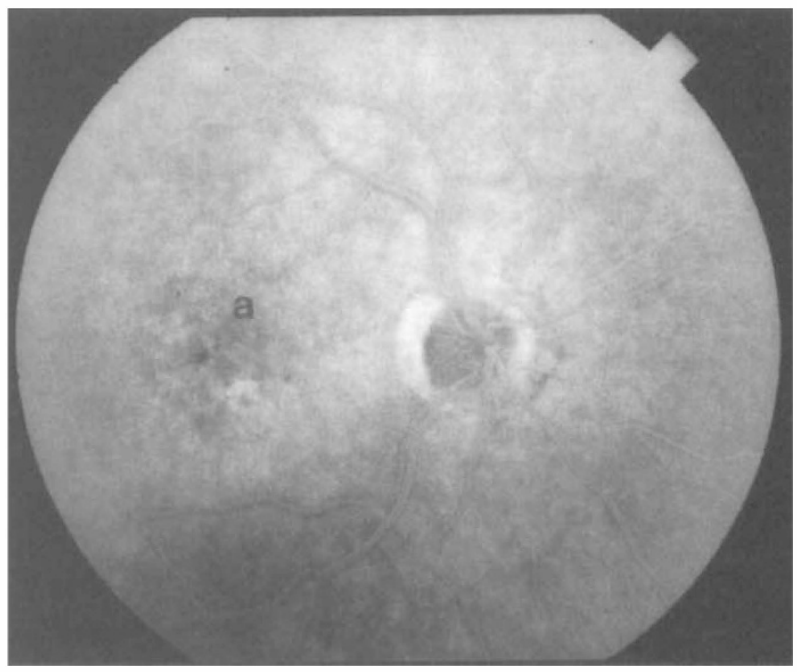

(B)

Fig. 2. (A) Fluorescein angiogram of the right fundus demonstrating hyperfluorescence due to leakage from the retinal artery macroaneurysm at the first bifurcation of the superotemporal retinal artery (open arrow). There is associated macular oedema ( $a$ ) and intra-retinal (b) and pre-retinal haemorrhage (c). (B) Fluorescein angiogram of the right fundus taken 10 weeks preceding that in (A), demonstrating some minimal hyperfluorescence at the macula suggestive of oedema (a) but no evidence of leakage from a retinal artery macroaneurysm or retinal haemorrhage.

superotemporal artery may be due to the fact that aneurysms in this location lead to more visual symptoms. ${ }^{2,4-6}$

Previous small non-randomised trials have been inconclusive regarding the benefits of laser treatment of this rare condition. ${ }^{8}$ Nevertheless the clinical implication is that recognition of the signs of generalised retinal arterial damage including exudation, arteriovenous nipping and hyaline vascular change in elderly hypertensive patients requires careful examination including fluorescein angiography to detect focal areas of retinal damage and regular follow-up.

\section{References}

1. Robertson DM. Macroaneurysms of the retinal arteries. Ophthalmology 1973;77:55-67.

2. Rabb MF, Gagliano DA, Teske MP. Retinal artery macroaneurysms. Surv Ophthalmol 1988;33:73-96.

3. Cleary PE, Kohner EM, Hamilton AM, Bird AC. Retinal macroaneurysms. Br J Ophthalmol 1975;59:355-61.

4. Lewis RA, Norton EWD, Gass JDM. Acquired arterial macroaneurysms of the retina. Br J Ophthalmol 1976;60:21-30.

5. Palestine AG, Robertson DM, Goldstein BG. Macroaneurysms of the retinal arteries. Am J Ophthalmol 1982;93:164-71.

6. Giuffre G, Montalto PF, Amodei G. Development of an isolated retinal macroaneurysm of the cilioretinal artery. Br J Ophthalmol 1987;71:445-8.

7. Leishman $R$. The eye in general vascular disease, hypertension and arteriosclerosis. Br J Ophthalmol 1957;41:641-701.

8. Brown DM, Sobol WM, Folk JC, Weingeist TA. Retinal arteriolar macroaneurysms: long term visual outcome. $\mathrm{Br} \mathrm{J}$ Ophthalmol 1994;78:534-8.

M.T. Cahill

D.I. Flitcroft

R.W. Acheson

The Institute of Ophthalmology

University College Dublin

Mater Misericordiae Hospital

Eccles Street

Dublin 7
Republic of Ireland

Tel: 35318600254

Fax: 35318305693

e-mail: mcahill@indigo.ie

Sir,

\section{Bilateral iris sector heterochromia with or without}

\section{Hirschsprung's disease}

Hirschsprung's disease (HSCR) is a relatively common congenital disease of panethnic distribution. It is also known as congenital aganglionosis megacolon, with absence of intrinsic ganglion cells in the distal gastrointestinal tract leading to intestinal obstruction in the neonatal stage. ${ }^{1}$ The presence of HSCR in ophthalmologic discorders such as Waardenburg's syndrome has been documented. ${ }^{2}$ In one early report, a patient with Waardenburg's syndrome had bicoloured irides, megacolon and deafness. ${ }^{3}$ Association of HSCR with just iris sector heterochromia without ocular problem is rare and has only been reported twice previously as a discrete disease entity of neural crest syndrome. ${ }^{4}$ One report was on a Mexican family that had three young siblings manifesting the syndrome. One other male sibling had depigmented unilateral iris spot and no HSCR. Both parents and three other siblings were unaffected. This family implied an autosomal recessive mode of inheritance. Another report was on a 6-monthold female infant of Asian Indian origin and no other family member was affected..$^{5}$ The link between iris heterochromia and HSCR is likely to be their common origin from neural crest. Both involve insufficient or defective neural crest cells, the former for the development of pigment epithelium of the iris and the latter aganglionic segments usually affecting the distal large bowel. ${ }^{6,7}$ 